\title{
Liquid Crystal Polymer (LCP): A New Organic Material for the Development of Multilayer Dual-Frequency/Dual-Polarization Flexible Antenna Arrays
}

\author{
G. DeJean, R. Bairavasubramanian, D. Thompson, G. E. Ponchak, M. M. Tentzeris, and J. Papapolymerou
}

\begin{abstract}
The development of dual-frequency (14 and $35 \mathrm{GHz}$ ), dual-polarization microstrip antenna arrays is presented for the first time on liquid crystal polymer (LCP) multilayer technology. Some of the properties of LCP, such as multilayer (three-dimensional) vertical integration capability, good electrical and mechanical properties, and near-hermetic nature, make this substrate a practical choice for the design of low-cost antenna arrays that can be integrated with remote sensing applications operating in the $\mathrm{Ku}$ and millimeter-wave frequency bands. This work illustrates the potential of LCP as a low-cost, "all-package" solution for developing compact, flexible, antenna arrays that can be used in future communication and remote sensing systems.
\end{abstract}

Index Terms-Dual frequency/dual polarization, liquid crystal polymer, microstrip antenna array, multilayer fabrication.

\section{INTRODUCTION}

M ANY radar and communication systems need antennas with dual-polarization and dual-frequency capabilities for higher capacity data transfer. Microstrip patch antennas are often desirable antenna elements in such applications due to their low cost, low profile, light weight, and ease of fabrication characteristics [1]. In recent years, there has been much research done in the field of designing dual-frequency and dual-polarization microstrip antenna arrays [2]-[5].

When designing dual-frequency, dual-polarized microstrip antenna arrays, one has to confront many parameters of interest and the associated complexity both in design and fabrication. There is a need for a complex feeding structure that minimizes interconnect loss, feedline radiation, and crosscoupling [6]. Substrate thickness can affect cross-polarization levels as well as bandwidth and efficiency. The distance of the antenna elements in the array can affect $-3-\mathrm{dB}$ beamwidth, directivity, and side-lobe levels besides impacting the overall size. Careful consideration needs to be given to avoid crosscoupling between the antenna arrays operating at different frequencies, blockage effects, and edge diffraction [7]. It is almost impossible to

Manuscript received October 1, 2004; revised November 29, 2004. This work was supported by National Aeronautics and Space Administration under Contract NCC3-1015.

G. DeJean, R. Bairavasubramanian, D. Thompson, M. M. Tentzeris, and J. Papapolymerou are with the Georgia Institute of Technology, Atlanta, GA 30318 USA (e-mail: gdejean@ece.gatech.edu; gtg237c@prism.gatech.edu).

G. E. Ponchak is with the NASA Glenn Research Center, Cleveland, OH 44135 USA.

Digital Object Identifier 10.1109/LAWP.2004.841626 achieve all the aforementioned performance with a single layer structure. A multilayer architecture is required that can also result in very compact implementations. One such design of a dual-frequency, dual-polarized microstrip antenna array incorporating vertical integration was proposed by Granholm and Skou [8]. This design consists of C-band and L-band patches operating at 1.25 and $5.3 \mathrm{GHz}$, respectively, on the metal layers separated by substrate layers of three distinct dielectric media including foam.

Although there have been many reported examples of dual-frequency, dual-polarization microstrip antenna arrays on substrates, such as Duroid, these designs are not always favorable for a radio frequency (RF) system-on-a-package (SOP) low-cost technology due to various undesirable substrate properties. Materials, like Duroid, are often used in conjunction with low dielectric constant foam to realize multilayer configurations. Such composite multilayer structures are subjected to greater stress due to coefficient of thermal expansion (CTE) mismatches, which can alter the dimensions of the structure. There are many other thermal and mechanical problems inherent in such a multilayer design formed by integrating different materials. In order to overcome these problems and to integrate switches and phase shifters to enhance the reconfigurability, thus taking full advantage of polarization diversity and beam-scanning capabilities, there is a need for a laminated substrate that has vertical integration capabilities and is suitable for packaging RF passive and active components and embedded functions.

Liquid crystal polymer (LCP) offers such an unique combination of properties. This material has gained much consideration as a potential high performance microwave substrate and packaging material recently [9]-[11]. Its low dielectric constant and low loss tangent [12] in tandem with low water absorption coefficient [13] and low cost make it a first choice material for developing multilayer antenna arrays. Additionally, its CTE can be adjusted through thermal treatments, facilitating integration of integrated circuits in SOP modules. Furthermore, LCP, being a flexible material, leads to convenient deployment of antennas in space. Large sheets of LCP containing antenna arrays can be flexed, rolled up, and easily deployed.

This paper presents for the first time, the design, fabrication, and measurement of dual-frequency (14 and $35 \mathrm{GHz}$ ), microstrip antenna arrays with dual-polarization capabilities 


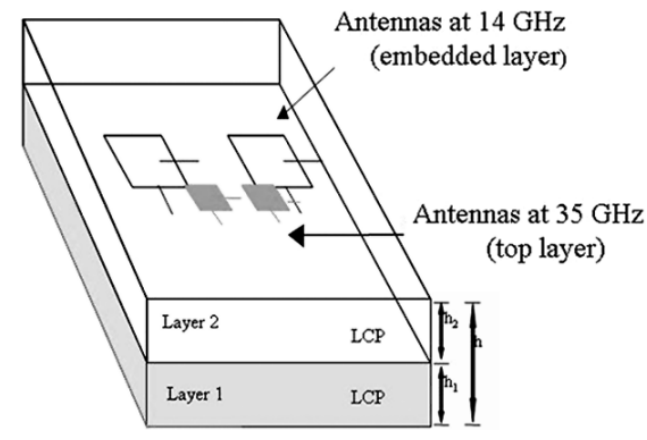

Fig. 1. Multilayer antenna array architecture; $h_{1}=8$ mils, $h_{2}=9$ mils, $h=17$ mils.

excited separately at each frequency on flexible LCP multilayer substrates. These designs can eventually be applied to the remote sensing of precipitation at 14 and $35 \mathrm{GHz}$, respectively. They can be extended by integrating RF microelectromechanical systems (MEMS) switches [14] with the antenna arrays to switch polarizations, introducing the possibility of a low-power reconfigurable antenna array design. Full-wave simulations of the antenna array designs were performed and validated with good agreement of measured results of scattering parameters and radiation patterns.

\section{ARRAY DESIGN}

The generic multilayer architecture of the dual-polarization, dual-frequency microstrip antenna array at 14 and $35 \mathrm{GHz}$, respectively, is shown in Fig. 1. The total substrate thickness $h$ for the design is 17 mils, consisting of two LCP layers (each 8 mils thick) and a 1-mil bonding layer. The $14-\mathrm{GHz}$ antenna array is embedded on an 8 mil layer $h_{1}$ while the $35-\mathrm{GHz}$ patches that are physically smaller are placed on the top layer (at the interface of LCP and air) to minimize blockage of the 14-GHz radiation. The particular choice of substrate thicknesses stemmed from extensive analysis of their influence on cross-polarization levels, bandwidth, and efficiency at each frequency. The feed network for each array was placed in the same layer as the radiating elements. This configuration was chosen ahead of many other configurations, including aperture-coupled and proximity-coupled feeding, to minimize computational and fabrication complexity.

The top view of the dual-frequency antenna array with diagonal patch elements is shown in Fig. 2. This particular design is intended for precipitation radar systems wherein similar characteristics for orthogonal polarizations and polarization purity are of utmost importance, no matter what frequency band is used [15]. As shown in the figure, the patches are rotated by $45^{\circ}$ and the polarization directions are at $45^{\circ}$ and $135^{\circ}$ as opposed to the traditional $\mathrm{x}-\mathrm{y}$ directions. This arrangement helps in realizing a symmetrical feed network for both polarizations with similar impedance and radiation pattern characteristics. As a first modeling step, the switching of polarizations is controlled by the presence of "hard-wired" perfect "short" and "open" conditions. In a practical implementation, RF MEMS switches will be used to switch polarizations and steer the main beam. To simplify the antenna structure, the design approach uses an unloaded 200- $\mu \mathrm{m}$ gap to simulate an "OFF" state and a

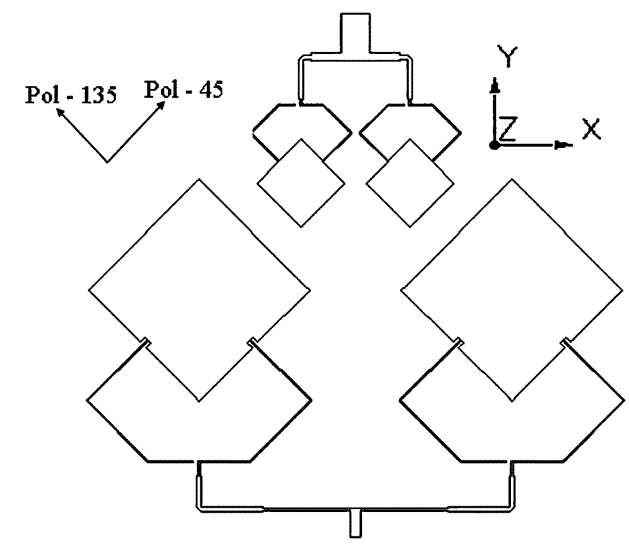

Fig. 2. Top view-Symmetrically fed array.

continuous feedline to simulate an "ON" state. In order to minimize the radiation effects of the feed lines, the lines that directly connect to the radiating element are made as thin as possible. A recessed patch feed and a combination of $\mathrm{T}$-junctions and quarter-wave transformers are employed to achieve better matching and a symmetric feed structure is used to expand into a $2 \times 1$ array. EmPicasso, a method-of-moments-based frequency domain full-wave solver, was used for design and simulations.

\section{FABRICATION/MEASUREMENT SETUP}

The antenna arrays were fabricated with two copper-clad 8-mil LCP dielectric sheets and one 1-mil LCP adhesion layer from Rogers Corporation. Although a thick copper layer may restrict the minimum feature size due to undercut problems, it is difficult to sputter/electroplate thin layers of copper on LCP reliably because it has a low stiction coefficient to copper. Therefore, thick copper cladding is used and the etch process was characterized and the patterns are modified beforehand to compensate for the undercut. The overetch is approximately $13 \mu \mathrm{m}$ for $18-\mu \mathrm{m}$-thick copper layer and is significant compared to the width of the thin feedlines $(50 \mu \mathrm{m})$ that are connected to the patches. Besides it can alter critical dimensions of the array, such as the patch length and width. This can cause undesirable shifts in the resonant frequency of the array especially at $35 \mathrm{GHz}$. An alternative to using thick copper would be to introduce a thin seed layer such as titanium between copper and LCP to improve stiction. Although this was not tried, a thin seed layer $(0.3 \mu \mathrm{m})$ will have no effect on the array performance because the copper layer $(3 \mu \mathrm{m})$ will be much thicker in comparison. Such layers are often used in semiconductor circuits with no effect on performance. Shipley 1827 photoresist was used for pattern definition and the arrays are exposed under 16000-dpi mask transparencies pressed into sample contact with 5-in glass mask plates. Photoresist development and a wet chemical etch with ferric chloride were then performed to complete the antenna patterning. The LCP layers with the 14and the $35-\mathrm{GHz}$ array were then bonded together in a Karl Suss SB-6 silicon wafer bonder using a 1-mil low melt LCP bond layer sandwiched between the two 8-mil high melt LCP core layers. The bond layer melts at a lower temperature than the core layers and its flow coupled with the tool pressure applied between the core layers results in the realization of multilayer 

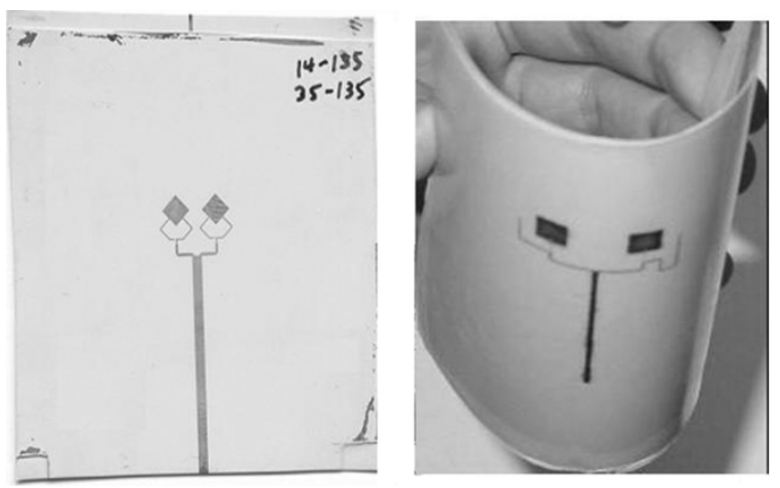

Fig. 3. Photograph of the fabricated symmetrically-fed array \& photo demonstrating flexibility.

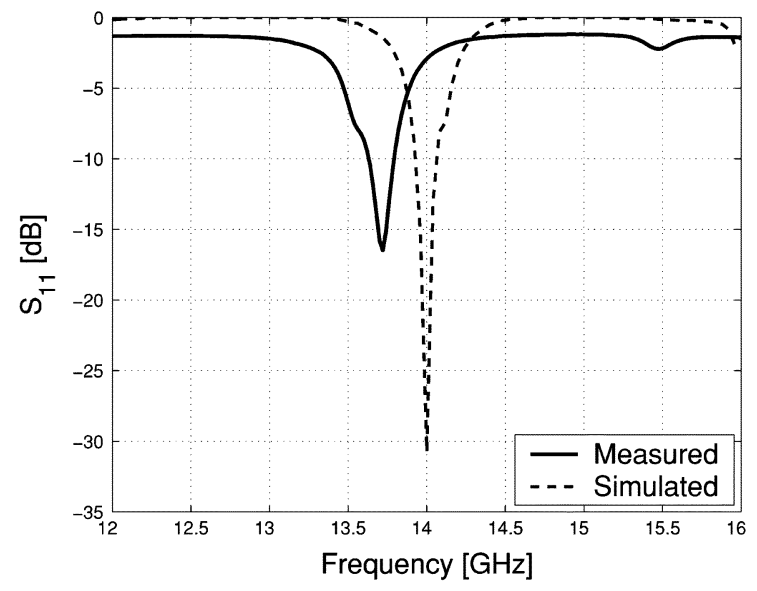

Fig. 4. Return loss-14-GHz array.

LCP structures. This is the most critical step in the fabrication process and has to be understood thoroughly to create multilayer LCP structures reliably. Several experiments were carried out to optimize the temperature and the tool pressure to achieve good bonding while preventing shrinkage, formation of bubbles, and melting of core layers. The bubbles can result in air gaps that can affect the array performance at millimeter-wave frequencies. Photographs of $2 \times 1$ antenna arrays on LCP showing the flexibility of the substrate are shown in Fig. 3.

The array was mounted on an aluminum fixture that included a 2.4-mm coaxial-to-microstrip connector to measure the return loss of the array. A short-, open-, load-, and- thru- [16] calibration was performed on a vector network analyzer with the reference planes at the end of the coaxial cables. When required, the microstrip launcher was adjusted to improve the antenna under test (AUT) to coaxial launcher impedance match. An anechoic chamber with the AUT as the receive element and a $15-\mathrm{dB}$ gain horn antenna as the transmitting antenna was used for radiation pattern measurements. The AUT was rotated through the measurement plane, and the entire system, including the data recording, was automated. Because the microstrip launcher and the absorbing material placed around it covered a portion of the plane during the scan, there was a slight asymmetry in the radiation patterns due to the characterization system. In addition,

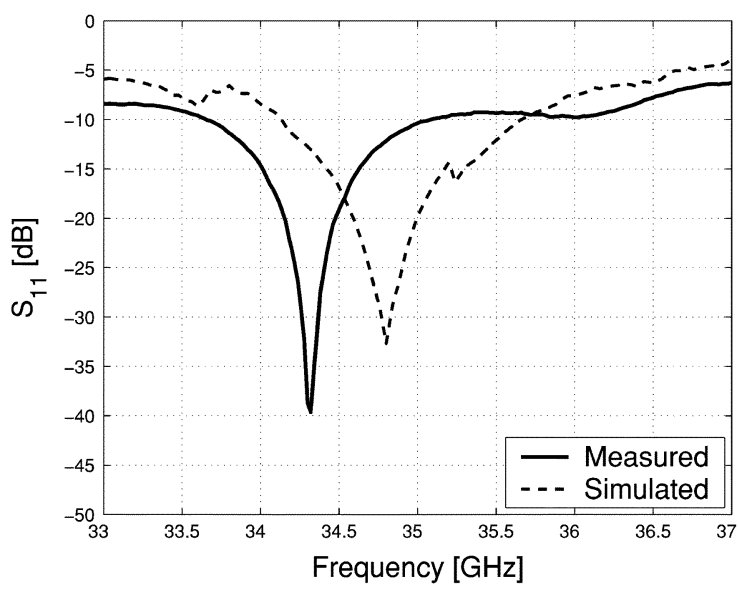

Fig. 5. Return loss-35-GHz array.

TABLE I

RETURN LOSS CHARACTERISTICS OF THE 14-GHz ARRAY

\begin{tabular}{c||c||c}
\hline \multicolumn{1}{c||}{ Characteristic } & Simulated 14-135 & Measured 14-135 \\
\hline Resonant Frequency & $14 \mathrm{GHz}$ & $13.72 \mathrm{GHz}$ \\
\hline Return Loss & $-30.7 \mathrm{~dB}$ & $-16.5 \mathrm{~dB}$ \\
\hline$-10 \mathrm{~dB}$ Return Loss Bandwidth & $140 \mathrm{MHz}$ & $160 \mathrm{MHz}$ \\
\hline Percent Bandwidth & $1.00 \%$ & $1.17 \%$ \\
\hline
\end{tabular}

TABLE II

RETURN LOSS CHARACTERISTICS OF THE 35-GHz ARRAY

\begin{tabular}{c||c||c}
\hline \multicolumn{1}{c||}{ Characteristic } & Simulated 35-135 & Measured 35-135 \\
\hline Resonant Frequency & $34.87 \mathrm{GHz}$ & $34.32 \mathrm{GHz}$ \\
\hline Return Loss & $-32.5 \mathrm{~dB}$ & $-39.6 \mathrm{~dB}$ \\
\hline -10 dB Return Loss Bandwidth & $1560 \mathrm{MHz}$ & $1530 \mathrm{MHz}$ \\
\hline Percent Bandwidth & $4.47 \%$ & $4.46 \%$ \\
\hline \multicolumn{2}{|c|}{} \\
\hline
\end{tabular}

the absorber affected the radiation pattern at scan angles greater than $70^{\circ}$ off boresight.

\section{RESULTS}

The simulated and measured return loss plots versus frequency are shown in Figs. 4 (at $14 \mathrm{GHz}$ ) and 5 (at $35 \mathrm{GHz}$ ). The results are for the $135^{\circ}$ polarization though they are the same for the $45^{\circ}$ polarization also owing to the symmetric arrangement. The dual-frequency array was excited at one frequency, while the other array was treated as a parasitic element. The results are summarized in Tables I and II. The shift in the resonant frequency can be attributed to fabrication tolerances. The discrepancy in return loss at $14 \mathrm{GHz}$ is due to the extension of the feedline of the embedded ( $14 \mathrm{GHz})$ antenna to a point where the top laminated layer of the substrate no longer covers the feedline, thus, modifying its characteristic impedance. The measured impedance bandwidths at both frequencies are in good agreement with those of the simulated designs.

Additionally, the simulated and measured two-dimensional radiation patterns are shown in Figs. 6 and 7 for the $E$ - and $H$-plane at $14 \mathrm{GHz}$, respectively, and Figs. 8 and 9 for the $E$ - and $H$-plane at $35 \mathrm{GHz}$, respectively. The results are summarized in Tables III and IV. The $E$-plane and $H$-plane beamwidths and the shapes of the co-polarized patterns are consistent for both the simulated and measured patterns of the 


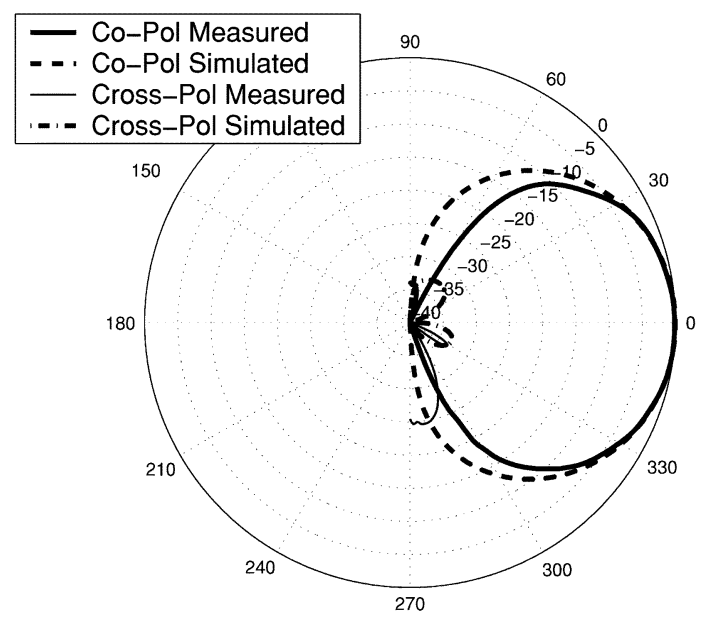

Fig. 6. E-plane radiation pattern-14-GHz array.

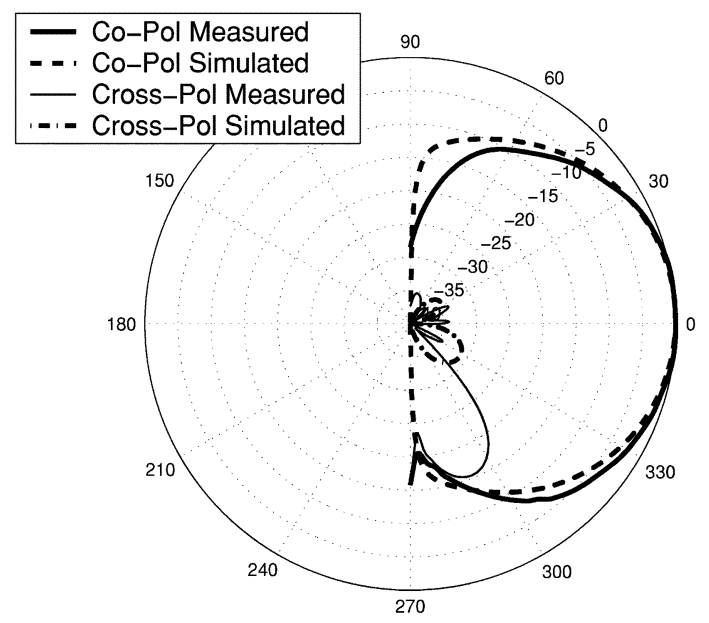

Fig. 7. $H$-plane radiation pattern-14-GHz array.

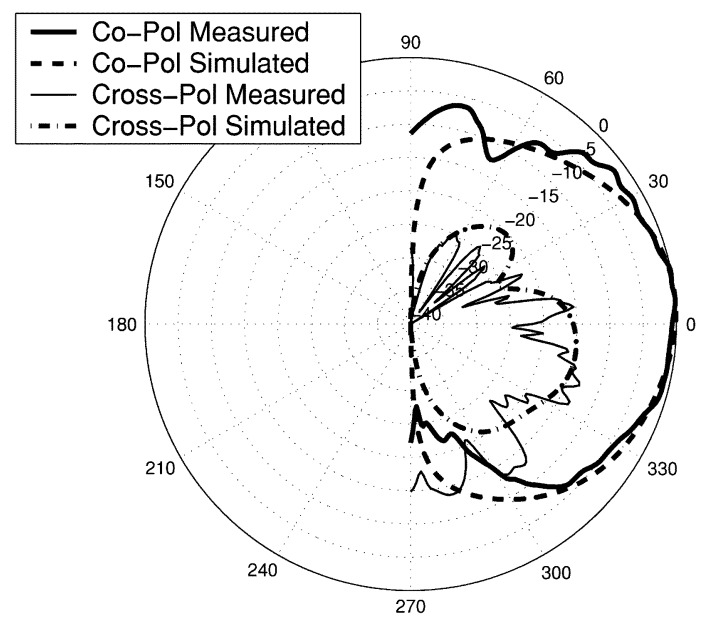

Fig. 8. E-plane radiation pattern-35-GHz array.

arrays which is expected for a symmetric configuration. The center-to-center distance can be increased to reduce the $E$-plane beamwidth to a value close to the $H$-plane beamwidth, but sidelobes will start to form as a result of this increase. The measured cross polarization levels also agree well with the predicted values for scan angles less than $70^{\circ}$. The discrepancy at angles

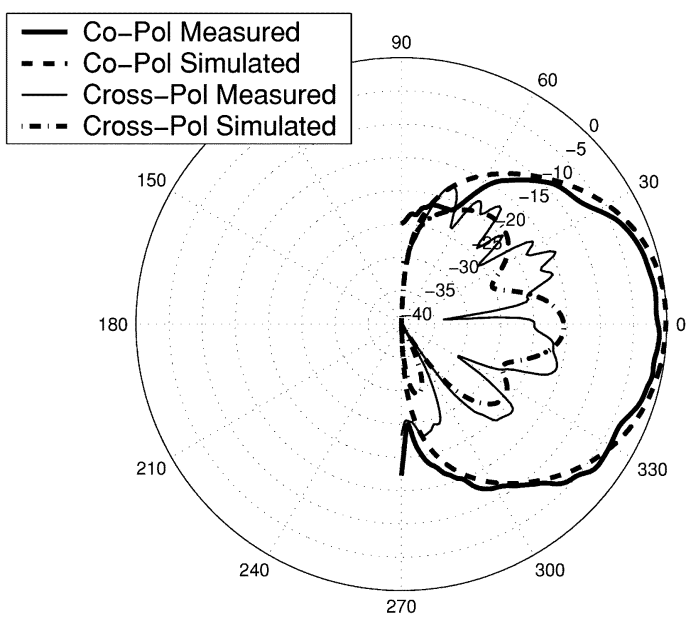

Fig. 9. $\quad H$-plane radiation pattern-35-GHz array.

TABLE III

RADIATION PATTERN CHARACTERISTICS OF THE 14-GHz ARRAY

\begin{tabular}{c||c||c}
\hline \multicolumn{1}{c||}{ Characteristic } & Simulated 14-135 & Measured 14-135 \\
\hline E-Plane -3 dB Beamwidth & $65^{\circ}$ & $67^{\circ}$ \\
\hline H-Plane -3 dB Beamwidth & $58^{\circ}$ & $58^{\circ}$ \\
\hline Max. Cross-pol.(E-plane) & $-31 \mathrm{~dB}$ & $-25 \mathrm{~dB}$ \\
\hline Max. Cross-pol.(H-plane) & $-33 \mathrm{~dB}$ & $-30 \mathrm{~dB}$ \\
\hline
\end{tabular}

TABLE IV

RADIATION PATTERN CHARACTERISTICS OF THE 35-GHz ARRAY

\begin{tabular}{c||c||c}
\hline \multicolumn{1}{c||}{ Characteristic } & Simulated 35-135 & Measured 35-135 \\
\hline E-Plane -3 dB Beamwidth & $65^{\circ}$ & $66^{\circ}$ \\
\hline H-Plane -3 dB Beamwidth & $59^{\circ}$ & $59^{\circ}$ \\
\hline Max. Cross-pol.(E-plane) & $-15 \mathrm{~dB}$ & $-14 \mathrm{~dB}$ \\
\hline Max. Cross-pol.(H-plane) & $-16 \mathrm{~dB}$ & $-15 \mathrm{~dB}$ \\
\hline
\end{tabular}

above $70^{\circ}$ is due to the presence of the absorber as explained in the measurement section. In addition, it has been noted in [1] and [17] that the cross-polarization level tends to increase as the substrate thickness increases. Therefore, the higher frequency $(35 \mathrm{GHz})$ antenna array on the electrically thicker substrate exhibits a worse cross-polarization level than the lower frequency $(14 \mathrm{GHz})$ array on the electrically thinner substrate. An improved design would place the $35-\mathrm{GHz}$ patches on a thinner (e.g., 4 mil) LCP substrate. To demonstrate the flexibility and mechanical stability of the multilayer LCP substrate, which is important for deployable antennas, antenna arrays were flexed several times and recharacterized. The return loss and radiation patterns were unchanged within the repeatability of the measurement equipment.

\section{CONCLUSION}

A dual-frequency (14 and $35 \mathrm{GHz}$ ), microstrip antenna array with dual-polarization capabilities excited separately at each frequency for SOP RF front-ends have been presented for the first time on flexible LCP multilayer substrate. The arrays exhibit a return loss of better than $15 \mathrm{~dB}$ in both frequency bands. The measured beamwidths were also in good agreement with the simulated results. The measured cross-polarization levels are higher than the predicted ones but can be improved by introducing a separate feed layer. The results shown here demonstrate the applicability of LCP for the development of 
low-cost, lightweight, and low-power RF front ends and antennas on an "all-package" solution for future communication and remote sensing systems operating up to millimeter-wave frequency ranges.

\section{REFERENCES}

[1] P. Bhartia, , I. Bahl, R. Garg, and A. Ittipiboon, Microstrip Antenna Design Handbook, 1st ed. Norwood, MA: Artech House, 2000.

[2] K.-L. Wong, S.-C. Pan, and C.-M. Chang, "Design of a two-element dual frequency microstrip array," in IEEE 1997 Antennas and Propagation Society Int. Symp. Dig., Montreal, QC, Canada, pp. 940-943.

[3] J.-H. Lu, "Broadband dual-frequency operation of circular patch antenna arrays with a pair of L-shaped slots," IEEE Trans. Antennas Propag., vol. 51, no. 5, pp. 1018-1023, May 2003.

[4] B. Lindmark, S. Lundgren, J. R. Sanford, and C. Beckman, "Dual-polarized array for signal-processing applications in wireless communications," IEEE Trans. Antennas Propag., vol. 46, no. 6, pp. 758-763, Jun. 1998.

[5] S. Gao and S. Zhong, "A dual-polarized microstrip antenna array with high isolation fed by coplanar network," in Proc. Radio and Wireless Conf., 1998 IEEE, Colorado Springs, CO, pp. 213-216.

[6] E. Levine, G. Malamud, S. Shtrikman, and D. Treves, "A study of the microstrip array antennas with feed network," IEEE Trans. Antennas Propag., vol. 37, no. 4, pp. 426-434, Jul. 1989.

[7] J. Huang, "The finite ground plane effect on the microstrip antenna radiation patterns," IEEE Trans. Antennas Propag., vol. 31, no. 4, pp. 649-653, Jul. 1983.

[8] J. Granholm and N. Skou, "Dual-frequency, dual-polarization microstrip antenna array development for high-resolution, airborne SAR," in Proc. Asia-Pacific Microwave Conf., Sydney, Australia, 2000, pp. 17-20.
[9] D. C. Thompson, O. Tantot, H. Jallageas, G. E. Ponchak, M. M. Tentzeris, and J. Papapolymerou, "Characterization of liquid crystal polymer (LCP) material and transmission lines on LCP substrates from 30-110 GHz," IEEE Trans. Microwave Theory Tech., vol. 52, no. 4, pp. 1343-1352, Apr. 2004.

[10] M. M. Tentzeris, J. Laskar, J. Papapolymerou, S. Pinel, V. Palazzari, R. Li, G. DeJean, N. Papageorgiou, D. Thompson, R. Bairavasubramanian, S. Sarkar, and J.-H. Lee, "3D Integrated RF and millimeter-wave functions and modules using liquid crystal polymer (LCP) system-on-package technology," IEEE Trans. Adv. Packag., vol. 27, no. 2, pp. 332-340, May 2004.

[11] V. Palazzari, S. Pinel, M. M. Tentzeris, L. Roselli, and J. Laskar, "A symmetrical dual-band WLAN filter in liquid crystal polymer system-onpackage technology," IEEE Microw. Compon. Lett., to be published.

[12] K. Jayaraj, T. E. Noll, and D. R. Singh, "RF characterization of a low cost multichip packaging technology for monolithic microwave and millimeter wave integrated circuits," in Proc. URSI Int. Symp. Signals, Systems, and Electronics, San Francisco, CA, 1995, pp. 443-446.

[13] B. Farrell and M. St. Lawrence, "The processing of liquid crystalline polymer printed circuits," in Proc. IEEE Electronic Components and Technology Conf., San Diego, CA, 2002, pp. 667-671.

[14] G. Wang, D. Thompson, M. Tentzeris, and J. Papapolymerou, "Low cost RF MEMS switches using LCP substrate," presented at the 34th European Microwave Conf., Amsterdam, The Netherlands, Oct. 2004.

[15] K. Bahadori and Y. Rahmat-Samii, "Ku/Ka bands precipitation radar antenna: Half-scale offset cylindrical reflector model [spaceborne]," in Proc. IEEE Antennas and Propagation Society Int. Symp., Columbus, OH, 2003, pp. 22-27.

[16] M. D. DuFault and A. K. Sharma, "A novel calibration verification procedure for millimeter-wave measurements," in IEEE MTT-S Int., Microwave Symp. Dig., vol. 3, Jun. 1996, pp. 1391-1394.

[17] T. Huynh, K. F. Lee, and R. Q. Lee, "Crosspolarization characteristics of rectangular patch antennas," Electron. Lett., vol. 24, no. 8, pp. 463-464, Apr. 1998 